\title{
Editorial for the Special Issue on Selected Papers from the "2019 International Symposium on Remote Sensing"
}

\author{
Fuan Tsai ${ }^{1, *(\mathbb{D})}$, Chao-Hung Lin ${ }^{2}{ }^{(}$, Walter W. Chen ${ }^{3}{ }^{(D)}$, Jen-Jer Jaw ${ }^{4}$ and Kuo-Hsin Tseng ${ }^{1(\mathbb{D})}$ \\ 1 Center for Space and Remote Sensing Research, National Central University, 300 Zhongda Road, Zhongli, \\ Taoyuan 32001, Taiwan; khtseng@csrsr.ncu.edu.tw \\ 2 Department of Geomatics, National Cheng-Kung University, No. 1, Daxue Road, East Dist., Tainan City 701, \\ Taiwan; linhung@mail.ncku.edu.tw \\ 3 Department of Civil Engineering, National Taipei University of Technology, No. 1, Sec. 3, \\ Chung-Hsiao E. Road, Taipei 10608, Taiwan; waltchen@ntut.edu.tw \\ 4 Department of Civil Engineering, National Taiwan University, No. 1, Sec. 4, Roosevelt Road, Taipei 10617, \\ Taiwan; jejaw@ntu.edu.tw \\ * Correspondence: ftsai@csrsr.ncu.edu.tw
}

Received: 15 June 2020; Accepted: 16 June 2020; Published: 17 June 2020

\begin{abstract}
The 2019 International Symposium on Remote Sensing (ISRS-2019) took place in Taipei, Taiwan from 17 to 19 April 2019. ISRS is one of the distinguished conferences on the photogrammetry, remote sensing and spatial information sciences, especially in East Asia. More than 220 papers were presented in 37 technical sessions organized at the conference. This Special Issue publishes a limited number of featured peer-reviewed papers extended from their original contributions at ISRS-2019. The selected papers highlight a variety of topics pertaining to innovative concepts, algorithms and applications with geospatial sensors, systems, and data, in conjunction with emerging technologies such as artificial intelligence, machine leaning and advanced spatial analysis algorithms. The topics of the selected papers include the following: the on-orbit radiometric calibration of satellite optical sensors, environmental characteristics assessment with remote sensing, machine learning-based photogrammetry and image analysis, and the integration of remote sensing and spatial analysis. The selected contributions also demonstrate and discuss various sophisticated applications in utilizing remote sensing, geospatial data, and technologies to address different environmental and societal issues. Readers should find the Special Issue enlightening and insightful for understanding state-of-the-art remote sensing and spatial information science research, development and applications.
\end{abstract}

\section{Introduction}

Since its inception in 1994, the International Symposium on Remote Sensing (ISRS) has become one of the prominent symposia on the photogrammetry, remote sensing and spatial information sciences. Starting in 2012, ISRS has been co-organized by the Korean Society of Remote Sensing (KSRS), Chinese (Taipei) Society of Photogrammetry and Remote Sensing (CSPRS) and Remote Sensing Society of Japan (RSSJ). The symposium is organized and held in Korea, Taiwan or Japan annually. The symposium not only serves as a scientific forum but also provides all the participants from all over the world with an effective platform for sharing and exchanging their new ideas and experiences with state-of-the-art techniques and the latest developments in research and development in the photogrammetry, remote sensing and spatial information sciences as well as their applications in related fields.

The 2019 International Symposium on Remote Sensing (ISRS-2019) was held in Taipei, Taiwan from April 17 to 19, 2019. There were 37 technical sessions, with a total of 226 papers presented in the symposium. The sessions covered a wide range of topics, which can be separated into several 
categories, such as sensors and platforms, algorithms and processing, spatial information, and remote sensing and interdisciplinary applications in different areas. In addition, as new sensing technologies and innovative artificial intelligence (AI) in conjunction with information and communication technology (ICT) have provided us with a great opportunity to tackle challenges in a broadening realm of research and applications, ISRS also organized sessions related to these emerging topics, such as Big Data, Deep Learning, the Internet of Things (IoT) and the like. Furthermore, ISRS-2019 also collaborated with the International Society for Photogrammetry and Remote Sensing (ISPRS) Working Group V/1 and V/8 to organize three workshops on "Open Source and Multi-level Education for Geospatial Technology" that were designed to introduce available open source data and software for geospatial analysis and applications to students, instructors and scientists.

Following its precedent [1], the organizer of the "2019 International Symposium on Remote Sensing" also planned a Special Issue in Remote Sensing to publish peer-reviewed feature papers presented at ISRS-2019. The submitted manuscripts were significantly extended from their original conference submissions. After rigorous review and revision, eight papers were selected for publication in the Special Issue on Selected Papers from the "2019 International Symposium on Remote Sensing".

\section{Overview of Contributions}

This Special Issue consists of featured contributions to the 2019 International Symposium on Remote Sensing held in Taipei, Taiwan, April 17-19, 2019. The topics of the selected papers cover a wide range of issues pertaining to the research and development in, and application and promotion of the remote sensing and spatial information sciences. The selected papers also describe innovative concepts, algorithms and applications with state-of-the-art geospatial sensors, systems, and data, in conjunction with emerging technologies such as artificial intelligence, machine learning, the internet of things, etc. A brief overview of the selected papers is given as follows.

\subsection{Radiometric Calibration of Optical Satellite Sensor}

Lin et al. [2] described two approaches to examine and calibrate the radiometric quality of an optical remote sensing instrument onboard the FORMOSAT- 5 satellite launched in 2017 by the National Space Organization (NSPO) of Taiwan. The described on-orbit measurement and calibration approaches employed the satellite signal in the solar spectrum (6S) radiative transfer model to estimate the surface reflectance, atmospheric effects, and path radiance for the radiometric intensity at the top of the atmosphere and used Landsat- 8 Operational Land Imager (OLI) data as a reference for validation and calibration.

The results described in the published paper reported a high consistency of the physical gains in spectra from cross-calibration filed experiments. However, the radiometric assessment and calibration also showed evident decay in the optical sensitivity of the sensor, thus suggesting a necessity of long-term radiometric calibration.

\subsection{Drought Assessment, Evapotraspiration Estimation and Land Surface Temperature (LST) Retrieval}

Yoon et al. [3] calculated and compared the evaporative stress index (ESI) derived from satellite-based remote sensing imagery to assess agriculture drought events in East Asian countries. Their study demonstrates that compared to vegetation indices, the ESI is a more effective indicator for short- and medium-term drought assessment using multi-spectral satellite images.

Similarly, Januar et al. [4] modified the spatial and temporal adaptive reflectance fusion model (STARFM) [5] to a spatial and temporal adaptive "emissivity" fusion model (STAEFM) in order to blend high-temporal resolution Himawari-8 satellite data with Landsat-8 thermal infrared images. Their fused data were then demonstrated to be capable of providing hourly evapotranspiration, dryness index, and land surface temperature (LST) information. 


\subsection{Machine Learning for Close-Range Photogrammetry and Image Analysis}

Machine learning-based algorithms have been rapidly adopted and adapted for remote sensing image processing and analysis, applied not only to conventional satellite and airborne images but also to data collected with mobile mapping systems and drones. Among the selected publications, Chuang and Sung [6] developed a mobile mapping platform that consists of low-cost infrared and depth cameras with micromachined microelectromechanical systems (MEMS) g-sensors (accelerometers) to support sewer pipeline inspection applications. The developed mobile mapping platform utilized the image POSE and simultaneous localization and mapping (SLAM) techniques to reconstruct 3D point clouds in indoor environments. The mask region-based convolutional neural network (Mask-RCNN) machine learning algorithm [7] was also adopted by their developed decision support platform for detecting defective pipeline areas.

Yang et al. [8] explored two neural network-based deep learning algorithms to segment color images collected using an unmanned aerial vehicle (UAV) for identifying lodged rice fields. The methodology and results described in their paper indicated that deep learning-based image segmentation (classification) outperformed the conventional Maximum Likelihood method, not only with a higher accuracy but also being 10 to 15 times faster.

\subsection{Integration of Remote Sensing and Spatial Analysis}

The amalgamation of remote sensing and spatial analysis is a powerful integration for sophisticated geospatial applications. On the one hand, remote sensing provides abundant sources of assorted data, while spatial analysis, on the other hand, maximizes the usefulness and practicability of these diversified data in various real-world applications.

In this regard, Gebru et al. [9] classified multi-temporal Landsat satellite images of the thematic mapper (TM) and Operational Land Imager (OLI) to identify land-use and land-cover (LULC) changes in Northern Ethiopia. The LULC changes were further analyzed to understand their impact on the hydrology of the dry Afromontane forest in the study area. The findings of their study revealed a high correlation between the land degradation and surface runoff and the LULC changes. Their study also demonstrates the usability and importance of remote sensing and spatial analysis in agro-ecological research and applications.

Principe and Takeuchi [10] collected and analyzed assorted geospatial data sets to evaluate the supply and demand of solar photovoltaic (PV) systems in the Asia Pacific region. The data used in their study include nighttime satellite images, digital surface models, land-cover data, road network data, and many other socio-economic data. These heterogeneous data were obtained from a variety of global or regional projects, space agencies, the United Nations, the World Bank, and other governmental and non-governmental organizations. The method presented in this paper first processed and integrated the collected data. Spatial analysis was then performed to assess the demand and supply of PV systems for identifying suitable areas for installing PV systems and estimating solar PV power output. In addition, a method was also proposed to extract power transmission lines from global road network and nightlight satellite data.

Last, but not least, Lin et al. [11] presented a Volunteered Geographic Information (VGI) platform to detect flooded areas and determine water levels based on the images acquired using the cameras of the VGI participants' smartphones. In their developed VGI system, digital image processing and a photogrammetric method were implemented to identify (classify) inundated areas and to estimate the flooding water level from the images uploaded by volunteers. Their examples demonstrated that the VGI images can be used as a reliable and effective source for the detection and monitoring of flooding by the disaster management authorities. 


\section{Results}

The selected papers published in this Special Issue highlight a variety of topics related to the photogrammetry, remote sensing and spatial information sciences, as well as their applications in different domains, presented in the "2019 International Symposium on Remote Sensing" (ISRS-2019). The platforms and systems described and discussed in the published papers include satellites, unmanned aerial vehicles (UAVs), ground-based mobile mapping systems, and smartphones, while the data utilized cover a wide spectrum and have assorted characteristics. The algorithms and methodology presented in the selected papers deal with diverse issues, from on-orbit radiometric calibration, data fusion, feature extraction, change detection and integrated spatial analysis, to state-of-the-art machine learning and deep learning image analysis. In particular, the sophisticated and diverse applications demonstrated and discussed in the published papers further signify the advantages and benefits of the remote sensing and spatial information sciences and their contributions to scientific, environmental and societal improvements, like the other papers presented at ISRS-2019.

Acknowledgments: We would like to thank all the authors who contributed to ISRS-2019 and this Special Issue and the staff in the editorial office.

Conflicts of Interest: The authors declare no conflict of interest.

\section{References}

1. Jung, H.-S.; Ryu, J.-H.; Park, S.-E.; Lee, H.; Park, N.-W. Special Issue on Selected Papers from the “International Symposium on Remote Sensing 2018". Remote Sens. 2019, 11, 1439. [CrossRef]

2. $\quad$ Lin, T.-H.; Chang, J.-C.; Hsu, K.-H.; Lee, Y.-S.; Zeng, S.-K.; Liu, G.-R.; Tsai, F.; Chen, H.-P. Radiometric Variations of On-Orbit FORMOSAT-5 RSI from Vicarious and Cross-Calibration Measurements. Remote Sens. 2019, 11, 2634. [CrossRef]

3. Yoon, D.-H.; Nam, W.-H.; Lee, H.-J.; Hong, E.-M.; Feng, S.; Wardlow, B.D.; Tadesse, T.; Svoboda, M.D.; Hayes, M.J.; Kim, D.-E. Agricultural Drought Assessment in East Asia Using Satellite-Based Indices. Remote Sens. 2020, 13, 444. [CrossRef]

4. Januar, T.W.; Lin, T.-H.; Huang, C.-Y.; Chang, K.-E. Modifying an Image Fusion Approach for High Spatiotemporal LST Retrieval in Surface Dryness and Evapotranspiration Estimations. Remote Sens. 2020, 12, 498. [CrossRef]

5. Gao, F.; Masek, J.; Schwaller, M.; Hall, F. On the blending of the Landsat and MODIS surface reflectance: Predicting daily Landsat surface reflectance. IEEE Trans. Geosci. Remote Sens. 2006, 44, 2207-2218.

6. Chuang, T.-Y.; Sung, C.-C. Learning and SLAM Based Decision Support Platform for Sewer Inspection. Remote Sens. 2020, 12, 968. [CrossRef]

7. He, K.; Gkioxari, G.; Dollár, P.; Girshick, R. Mask R-CNN. In Proceedings of the 2017 IEEE International Conference on Computer Vision (ICCV), Venice, Italy, 22-29 October 2017; pp. 2980-2988. [CrossRef]

8. Yang, M.-D.; Tseng, H.-H.; Hsu, Y.-C.; Tsai, H.P. Semantic Segmentation Using Deep Learning with Vegetation Indices for Rice Lodging Identification in Multi-date UAV Visible Images. Remote Sens. 2020, 12, 633. [CrossRef]

9. Gebru, B.M.; Lee, W.-K.; Khamzina, A.; Lee, S.-G.; Negash, E. Hydrological Response of Dry Afromontane Forest to Changes in Land Use and Land Cover in Northern Ethiopia. Remote Sens. 2019, 11, 1905. [CrossRef]

10. Principe, J.; Takeuchi, W. Supply and Demand Assessment of Solar PV as Off-Grid Option in Asia Pacific Region with Remotely Sensed Data. Remote Sens. 2019, 11, 2255. [CrossRef]

11. Lin, Y.-T.; Yang, M.-D.; Han, J.-Y.; Su, Y.-F.; Jang, J.-H. Quantifying Flood Water Levels Using Image-Based Volunteered Geographic Information. Remote Sens. 2020, 12, 706. [CrossRef] 